\section{Discontinuation of Canakinumab following Clinical Disease Remission Is Feasible in Patients with Systemic Juvenile Idiopathic Arthritis}

\section{To the Editor:}

The optimal duration of treatment in patients with juvenile idiopathic arthritis, especially systemic JIA (sJIA), is a major concern for parents and physicians alike. Innovative medicines have managed to control this otherwise potentially life-threatening condition ${ }^{1}$. However, questions remain regarding the duration and the best timing for cessation of treatment.

We report the results of a retrospective study describing the longterm outcome of children with sJIA treated with the antiinterleukin (IL)- $1 \beta$ monoclonal antibody canakinumab. In these children, treatment was discontinued following sustained clinical remission; we aim to identify potential relapse-associated risk factors.

This was a single-center retrospective case study conducted from January 2008 to January 2017 and including patients with sJIA (based on International League of Associations for Rheumatology criteria) ${ }^{2}$ who had clinically inactive sJIA (clinical remission on medication/Wallace criteria) ${ }^{3}$ for at least 2 years with canakinumab. Patients were monitored for up to 4 years [median time $3.4 \mathrm{yrs}$, interquartile range (IQR) 2.4-3.7] following attempted canakinumab withdrawal (the study period began at the time of canakinumab withdrawal). Demographics, clinical, and laboratory variables were recorded at the study onset. We also collected data on previous and concomitant medication exposure as well as clinical outcome following canakinumab discontinuation (remission-flare/American College of Rheumatology criteria; data not shown in full) $)^{4}$. Data were analyzed using SPSS 19.0. Differences were tested with the Mann-Whitney U test and Fisher's exact test and a $\mathrm{p}$ value $<0.05$ was set as the threshold for statistical significance. Our study was approved by the P. \& A. Kyriakou Children's Hospital Ethics and Research Committee (Ap. number ES4/i-345/2016-05). Written informed consent was obtained by the parent or legal guardian.

In a total of 12 patients ( 7 girls) with inactive sJIA (median age $8.5 \mathrm{yrs}$, IQR 3.7-11.3), canakinumab treatment was discontinued. Median disease duration at the time of canakinumab discontinuation was 3.8 years (IQR 2.7-6.4). All patients had received $4 \mathrm{mg} / \mathrm{kg}$ canakinumab at monthly intervals for at least 2 years. Median duration of treatment was 2.8 years (IQR 2.2-3.1). Discontinuation was gradual (spacing - 1 dose at 6 weeks and a subsequent dose at 8 ) in $75 \%$ of patients. All patients were taking corticosteroids when canakinumab was started ( $<1 \mathrm{mg} / \mathrm{kg}$ prednisolone), and they were discontinued within 3 to 9 months. Ten out 12 patients (84\%) were taking methotrexate (MTX; mean dose: $12.5 \mathrm{mg} / \mathrm{m}^{2}$ ), one (8\%) was taking leflunomide $(20 \mathrm{mg})$, and one $(8 \%)$ cyclosporine $(1.5 \mathrm{mg} / \mathrm{kg})$. They continued to take the same dose of synthetic disease-modifying antirheumatic drugs (DMARD) during treatment with canakinumab. Eight out of 12 patients $(75 \%)$ had received at least 1 other biologic (b)DMARD before starting canakinumab: $2(17 \%)$ anti-tumor necrosis factor- $\alpha, 1(8 \%)$ abatacept, $2(17 \%)$ tocilizumab, and $3(25 \%)$ anakinra. Two out of the 12 patients $(12.5 \%)$ had received at least 2 biologics prior to canakinumab, while 1 patient (8\%) had received 3 biologics.

In $33 \%(4 / 12)$ of patients, canakinumab treatment was successfully stopped with no relapse. Out of these 4 patients (all taking MTX), 3 (75\%) subsequently managed to stop MTX and remain in drug-free clinical remission (> 2 yrs). Eight out of 12 patients (75\%) flared during the followup period. Median duration of remission following canakinumab withdrawal was 4.5 months (IQR 3.2-11.2). Six out of 8 patients $(75 \% ; 50 \%$ of the total) were restarted on canakinumab while a short course of steroids resolved the flare in the remaining 2 patients. Subgroup analysis showed that biologic-naive patients $[3 / 4(75 \%)$ vs $1 / 8(12.5 \%), p=0.03]$ and patients with shorter median disease duration at the time of canakinumab discontinuation (1.8 vs $3.4 \mathrm{yrs}, \mathrm{p}=0.04)$ had a higher chance of successful withdrawal. Patients who relapsed were more likely to do so within 6 months of canakinumab discontinuation; $7 / 8(87.5 \%)$ relapsed in $<6$ months versus $1 / 8(12.5 \%)$ in more than 6 months $(p=0.02)$. The relapse percentages were identical in the abrupt (62\%) versus gradual discontinuation groups (67\%; $\mathrm{p}=0.07$, data not shown). Age $(\mathrm{p}=0.09)$ and $\operatorname{sex}(\mathrm{p}=0.08)$ did not affect clinical outcome (Table 1).

This was a retrospective analysis regarding discontinuation of canakinumab in patients with clinically inactive sJIA. In general, biologic-naive patients demonstrate a swift and sustained response to IL-1 blockade ${ }^{5}$. Moreover, while multiple studies have shown clinical benefit of IL-1-targeted drugs such as recombinant IL-1Ra, not all patients responded equally well ${ }^{6,7}$; nonetheless in our study $3 / 12$ patients who did not respond to IL-Ra responded to canakinumab. Moreover, the protocols in the aforementioned trials did not include a plan for tapering or stopping the drug once remission had been achieved ${ }^{8}$. On the other hand, a recently published study testing a stop strategy in patients with disease in remission at 3 months and at 2 years after the start of canakinumab showed promising results ${ }^{9}$. In accordance with other studies, timely initiation of an effective bDMARD appeared to be of importance in acquiring disease remission; the concomitant use of synthetic DMARD may be helpful in the effective withdrawal of bDMARD, but the numbers in our study were too small to draw a firm conclusion ${ }^{10}$. Our study demonstrated that canakinumab withdrawal is feasible in a proportion of patients with sJIA. Patients with shorter disease duration and no previous exposure to a bDMARD showed a higher chance of attaining longterm remission. Nonetheless, the required period of time in clinical disease remission while receiving canakinumab treatment prior to attempting discontinuation of the drug is still unclear.

DESPOINA N. MARITSI $\odot$, MD, PhD, MRCPCH, Infectious Diseases, Immunology and Rheumatology Unit, Second Department of Paediatrics, P. \& A. Kyriakou Children's Hospital, Medical School, National and Kapodistrian University of Athens, and Pediatric Faculty, Medical School, University of Cyprus, Nicosia, Cyprus; OLGA VOUGIOUKA, MD, PhD, Infectious Diseases, Immunology and Rheumatology Unit, Second Department of Paediatrics, P. \& A. Kyriakou Children's Hospital, Medical School, National and Kapodistrian University of Athens, Athens, Greece; DESPINA ELEFTHERIOU 10 , MD, PhD, MRCPCH, UCL-Great Ormond Street-Institute of Child Health, Infection, Immunity, Inflammation and Physiological Medicine, London, UK. Address correspondence to Dr. D. Maritsi, Infectious Diseases, Immunology and Rheumatology Unit, Second Department of Paediatrics, P. \& A. Kyriakou Children's Hospital, Medical School, National and Kapodistrian University of Athens, Athens, Greece. E-mail: dmaritsi@gmail.com

Table 1. Clinical characteristics of patients with sJIA associated with the ability to discontinue canakinumab.

\begin{tabular}{|c|c|c|c|}
\hline Characteristics & $\begin{array}{c}\text { Patients with sJIA } \\
\text { Who Flared }\end{array}$ & $\begin{array}{l}\text { Patients with sJIA } \\
\text { Who Remained } \\
\text { in Remission }\end{array}$ & $\mathrm{p}^{*}$ \\
\hline Male, 5 total, n (\%) & $3(43)$ & $2(40)$ & 0.1 \\
\hline Female, 7 total, n (\%) & $4(57)$ & $3(60)$ & \\
\hline $\begin{array}{l}\text { Previous non-canakinu } \\
\text { biologic use }\end{array}$ & 7/8 (87.5) & $1 / 8(12.5)$ & 0.03 \\
\hline $\begin{array}{l}\text { Disease duration at tim } \\
\text { discontinuation, yrs, } \\
\text { median (IQR) }\end{array}$ & of canakinumab & $1.8(1.3-3.4)$ & 0.04 \\
\hline $\begin{array}{l}\text { Age at time of canakin } \\
\text { yrs, median (IQR) }\end{array}$ & $\begin{array}{c}\text { mab discontinuation, } \\
8.9(7.8-12.7)\end{array}$ & $9.2(7.5-11.4)$ & 0.09 \\
\hline
\end{tabular}

* Fisher's exact test. sJIA: systemic juvenile idiopathic arthritis; IQR: interquartile range. 


\section{ACKNOWLEDGMENT}

The authors thank the families for their participation and support.

\section{REFERENCES}

1. Vastert SJ, Kuis W, Grom AA. Systemic JIA: new developments in the understanding of the pathophysiology and therapy. Best Pract Res Clin Rheumatol 2009;23:655-64.

2. Petty RE, Southwood TR, Manners P. International League of Associations for Rheumatology classification of juvenile idiopathic arthritis: second revision, Edmonton, 2001. J Rheumatol 2004;31:390-2.

3. Wallace CA, Huang B, Bandeira M, Ravelli A, Giannini EH Patterns of clinical remission in select categories of juvenile idiopathic arthritis. Arthritis Rheum 2005;52:3554-62.

4. Brunner HI, Lovell DJ, Finck BK, Giannini EH. Preliminary definition of disease flare in juvenile rheumatoid arthritis. J Rheumatol 2002;29:1058-64.

5. Vastert SJ, de Jager W, Noordman BJ, Holzinger D, Kuis W, Prakken BJ, et al. Effectiveness of first-line treatment with recombinant interleukin-1 receptor antagonist in steroid-naive patients with new-onset systemic juvenile idiopathic arthritis: results of a prospective cohort study. Arthritis Rheumatol 2014;66:1034-43.

6. Otten MH, Prince FH, Anink J, Ten Cate R, Hoppenreijs EP,
Armbrust W, et al. Effectiveness and safety of a second and third biological agent after failing etanercept in juvenile idiopathic arthritis: results from the Dutch National ABC Register. Ann Rheum Dis 2013;72:721-7.

7. Woerner A, Uettwiller F, Melki I, Mouy R, Wouters C, Bader-Meunier B, et al. Biological treatment in systemic juvenile idiopathic arthritis: achievement of inactive disease or clinical remission on a first, second or third biological agent. RMD Open 2015;1:e000036.

8. Ruperto N, Brunner HI, Quartier P, Constantin T, Wulffraat N, Horneff $\mathrm{G}$, et al. Two randomized trials of canakinumab in systemic juvenile idiopathic arthritis. N Engl J Med 2012;367:2396-406.

9. Ruperto N, Brunner HI, Quartier P, Constantin T, Wulffraat NM, Horneff $\mathrm{G}$, et al. Canakinumab in patients with systemic juvenile arthritis and active systemic features: results from the 5-year long-term extension of the phase III pivotal trials. Ann Rheum Dis 2018;77:1710-19.

10. Sota J, Insalaco A, Cimaz R, Alessio M, Cattalini M, Gallizzi R, et al. Drug retention rate and predictive factors of drug survival for interleukin-1 inhibitors in systemic juvenile idiopathic arthritis. Front Pharmacol 2019;9:1526.

First Release February 15 2020; J Rheumatol 2020;47:4; doi:10.3899/jrheum.190011 